\title{
An Improved In Vivo Angiogenesis Model of Chicken Chorioallantoic Membranes in Surrogate Shells Revealed the Pro-angiogenesis Effects of Chylomicrons
}

\author{
Xiao Wan, 1, 2, a, @, \& Zifu Zhang, 3, \& Lei Rui, 4 Rhodri S. Wilson, 2 Xiaoning Wu, 2 Xin Liu, 5
}

@ corresponding author, \& equal contributor

Vascular Cell. 2019; 11(1):1 | ( X Xiao Wan, Zifu Zhang, Lei Rui, Rhodri S. Wilson, Xiaoning Wu, Xin Liu Received: 29 November 2018 | Accepted: 13 February 2019 | Published: 11 March 2019

Vascular Cell ISSN: 2045-824X

DOI: https://doi.org/10.24238/13221-11-1-178

\author{
Author information \\ 1. Target Discovery Institute - University of Oxford; Oxford, Oxfordshire United Kingdom \\ 2. 2. Department of Oncology - University of Oxford; Oxford, Oxfordshire United Kingdom \\ 3. College of Animal Science and Veterinary Medicine - Xinyang Agriculture and Forestry University; Xinyang, Henan 464000, China \\ 4. Chinese University of Agriculture; Beijing, China \\ 5. Department of Pharmacy - Beijing Tiantan Hospital, Capital Medical University; Beijing, China
}

[a] xiao.pharma@gmail.com

\begin{abstract}
Chicken chorioallantoic membrane (CAM) models have been applied as an affordable and efficient angiogenesis model for many years in relevant areas such as cancer angiogenesis and cardiovascular sciences. However, this model has intrinsic weaknesses such as the difficulty of operation and the limited size of the viewing windows. Efforts of trying to improve this model, such as transferring the embryos into petri dishes, has led to compromised survival rate of the embryos. In this study we improve the workflow by using surrogate egg shells. The images obtained from the viewing windows of the surrogate shells allow us to apply automated image analysis. This CAM model in surrogate shells combined with automated image analysis significantly decreases the labour whilst also increasing the data quality, when compared to traditional CAM models. Furthermore, to demonstrate the potential of our model, we have investigated the effects of a range of chylomicrons, extracted from murine and human samples, using the improved CAM model, to seek clues to elucidate the controversial roles of high level chylomicrons (CMs) in hypertriglyceridaemia (HTG) on atherosclerosis. We show that both native and modified chylomicrons (CMs) promote in vitro and in vivo angiogenesis. As angiogenesis is a risk factor in atherosclerosis our results provide a new mechanism for the effect of hypertriglyceridaemia (HTG) on atherosclerosis development. In summary, we demonstrate an improved CAM model aided by automated image analysis, we demonstrate the potential of this model by demonstrating interfered angiogenesis caused by HTG and discuss the impact this could potentially cause other pathological processes, such as atherosclerosis or carcinogenesis. Furthermore, the significantly low cost of CAM models compared with traditional murine models for cardiovascular research will have potential 3Rs (replacement, reduction and refinement) significance for the research communities, especially for laboratories with limited budgets or regulatory restrictions.
\end{abstract}

\section{Keywords}

3Rs - in vivo angiogenesis assay - chicken chorioallantoic membrane assay - chylomicrons oxidative stress

\section{Glossary Terms}

Ctrl: control. Treated with PBS only; CM: chylomicrons; NC: native chylomicrons; Ox-LDL: oxidative LDL. 


\section{Introduction}

The chorioallantoic membrane (CAM) is a highly vascularised and multi-functional extraembryonic membrane found in birds and reptiles. The CAM has been established as a cost effective and technically robust model to study physiological processes related to angiogenesis [1]. When compared with mammalian based in vivo models the CAM model has unique advantages including significantly low cost, shorter experimental time and relatively low technical difficulty. Additionally, the CAM model has a unique place in research communities facing increasing demand for the replacement, reduction and refinement, known commonly as the 3Rs, of animals used in research. It has been accepted that CAM is not innervated, which allows no pain experienced by the chick embryo [2]. In our experience and despite the above advantages there still exists technical limitations for this model. One such limitation is that the window opening process can sometimes cause damage to the CAM structure, this in turn can cause the death of the embryos. Furthermore, the size and morphology of the windows are not easy to control, this causes limitations in the analysis following the image acquisition. The calculation of the blood vessel length and areas, which are routine in angiogenesis in vitro models, can be complex for the CAM images due to such factors as unequal sample illumination and variable pixel size. Other researchers have developed systems to in vitro culture avian embryos in artificial containers, such as petri dishes, however this typically leads to a lower survival rate [3]. To overcome these limitations researchers, including ourselves, have referred to using petri dish as a replacement for original egg shells for the chicken embryos, however, the survival rate of the embryos decreased. Research suggests that this decrease was not only caused by the removal of the embryos from their own shells, but also because of the lack of calcium in the artificial in vitro culture environment. In this study we have used Peking duck egg shells as a surrogate and have thoroughly tested and optimized the whole process.

One important application of the CAM model is the study of angiogenesis, the process of new capillary networks forming in new tissues [1, 2]. Angiogenesis has important roles in physiological tissue healing and remodeling and regulates pathological processes such as cancer and atherosclerosis development $[4,5,6]$. The formation of new blood vessels contributes to the tumour growth and metastasis of cancer cells, and the interactions between blood vessel cells such as endothelial cells and cancer cells remodel the tissue microenvironment for cancer cells [5]. In atherosclerosis newly formed blood vessels can be formed in advanced plaques, which is similar to that in a tumour, providing an unstable haemorrhagic rupture-prone environment [6]. To study the process of angiogenesis various in vitro models and in vivo models have been developed and applied. In vitro models include cultured primary endothelial cells such as human umbilical vein endothelial cells (HUVECS), which capitulates some basic morphology and function traits in endothelial dynamics in angiogenesis. However, it was found that in vitro angiogenesis models lack many vital aspects of the actual in vivo angiogenesis tissue microenvironment, such as the extracellular matrix to support the structure and growth of the vasculature system, the interstitial fluid to transport growth factors which constantly regulates the metabolism of the vascular cells and other cells such as immunological cells which actively communicate with endothelium to maintain the homeostasis of the whole system. As a result, in vivo models are used to confirm the pre-test results obtained from in vitro angiogenesis models. Examples of these models include Matrigel plug xenograft models in mice, corneal blood vessel formation model in rabbits, rat aortic ring model and CAM model [5].

To test the feasibility of this improved CAM assay in surrogate egg shells we used the model to test if chylomicrons can regulate angiogenesis. Chylomicrons (CMs) are one of the five major groups of lipoproteins and consist mainly of triglycerides (85-92\%). Excreted by intestines native CMs are produced in lymph microvasculature system, then experiences oxidative modification by vascular endothelial cells and are eventually up taken by lipoprotein lipase (LPL) in the liver [7]. Hypertriglyceridaemia (HTG) is defined as high blood levels of triglyceride and is associated with cancer and atherosclerosis. Aberrant lipoprotein profile has been found in cancer patients and the association with increased mortality has been suggested [8]. Lipid metabolism can reprogramme this phenotype in tumourigenesis and confers growth and survival advantage of different cancer types [9]. A recent study demonstrated that lipiddependent metabolic reprogramming is an alternative mechanism of anti-angiogenic drug resistance [10]. HTG is also associated with atherosclerosis development. However, the relationship between CMs and atherosclerosis is still controversial [11]. Some reports showed CMs had no contribution to the atherosclerosis process due to the large diameter of the particle, which does not allow CM influx to the intima and accelerate atherosclerosis [12], while data from our lab revealed that accumulation of CM may be proatherogenic due to vascular endothelium impairment caused by oxidative modification to $\mathrm{CM}$ [11]. The mechanism that links lipid metabolism to angiogenesis is still not clear. Regarding to the roles of abnormal lipoprotein profiles in atherosclerosis (AS), studies show that at low concentration, oxidised low density lipoprotein (ox-LDL) enhances angiogenesis, thus can be beneficial or rise the risk of thrombosis in different situations [13]. In this study, we used the improved CAM model to investigate the effects of CM on angiogenesis, another type of lipoprotein might provide a potential relationship between HTG and atherosclerosis, and some clue for controversies on the effect of $\mathrm{CM}$ on atherosclerosis development. 


\section{Materials and Methods}

\section{Cell Culture}

Human umbilical vein endothelial cells (HUVECS) were isolated and cultured according to the protocol we previously published [14]. Briefly, 0.1\% collagenase type I was used to isolate umbilical vein endothelial cells at $370 \mathrm{o}$ for 15 minutes. Passages 2-6 in exponential growth condition were used for experiments. For growth medium, M199 medium (Gibco) supplemented with $5 \mu \mathrm{g} / \mathrm{mL}$ VEGF (SigmaAldrich), $12.5 \mathrm{U} / \mathrm{mL}$ heparin (Sigma-Aldrich) and $20 \%$ fetal bovine serum (FBS, Gibco) are used.

\section{Isolation of Chylomicrons}

Native chylomicrons were isolated from rat mesenteric lymph duct according to previous reports $[9,15]$. Briefly, SD rats were aneathesized ( $2 \%$ phenol barbitol $1 \mathrm{~mL} / 200 \mathrm{~g}$ body weight) and lymph were collected by cut off the white lymphatic duct 2 hours after the gavage of olive oil $1 \mathrm{~mL} / 100 \mathrm{~g}$ body weight. Modified chylmicrons were collected from the plasma of GPIHBP1-/- mice according to protocols previously reported [15]. Briefly, the collected lymph or plasma are injected under the PBS and centrifuged by $25000 \mathrm{rpm}, 20 \mathrm{~min}$ at $100 \mathrm{C}$. Only the top white layer was collected and considered as chylomicrons. All the procedures in this study were performed according to animal welfare and ethics guidelines of Peking University Health Science Centre (permit number: LA 2010-061).

\section{ROS measurement}

Reactive oxygen species in HUVECs was probed by 2',7'-dichlorofluorescin diacetate (DCFH-DA, Fluka), which can be deacetylated by intracellular esterases to DCFH, and oxidized to highly fluorescent dichlorofluorescein (DCF) in the presence of intracellular ROS [14, 15]. $5 \mu \mathrm{M}$ DCFH prepared in PBS was used to incubate with HUVECs for 30 minutes at $370 \mathrm{C}$. Then the images were captured on a fluorescence microscope (Leica). The excitation wavelength for DCF is $492-495 \mathrm{~nm}$, and the emission wavelength is $517-527 \mathrm{~nm}$.

\section{Nitric oxide / Nitric oxide synthase ( NO/ NOS) measurements}

NO/NOS measurements were carried out using Griess reagent-based NO/NOS measurementkit (Jiancheng, Nanjing, China) and were used according to the manufacturer's instructions.

\section{Cell Proliferation Assay}

3-(4,5-Dimethylthiazoyl-2-yl)-2,5-diphenyltetrazolium bromide (MTT) assay was used to test the cell proliferation and viability [14]. HUVECs $(5 \times 104$ cells/well in 96-well plates) were treated by CMs of various concentrations for certain periods. Then the cells were rinsed with PBS for three times, added into MTT work solution ( $0.5 \mathrm{mg} / \mathrm{mL}$ in PBS) and incubated for another 4 hours. Then the medium was aspirated and the formazan crystals formed were dissolved in $200 \mu \mathrm{L}$ dimethyl sulfoxide (DMSO) and the absorbance at $490 \mathrm{~nm}$ was measured using a microplate reader (Sunrise).

\section{Chick chorioallantoic membrane (CAM) angiogenesis assay in surrogate shells}

All the procedures in this study were performed according to animal welfare and ethics guidelines of China Agricultural University (permit number: SYXK 2013-0013). Chicken chorioallantoic membrane assay in surrogate egg shells was developed by us based on previous protocols [3]. Fertilized chicken eggs (SCXK Jing 20050001, Beijing Merial Vital Experimental Animal Technology Co., LTD) were firstly rinsed with $0.1 \%$ benzalkonium chloride, then sterilized by spraying $70 \%$ ethanol solution. After the shells were dried the eggs were kept at an incubator (Beijing Haijiang Incubation Equipment Ltd. China) set at $37 \circ \mathrm{C}$, humidity $55-65 \%$ at $90 \circ \mathrm{C}$. The eggs were turned over every two hours. After 5 days of incubation, $70 \%$ ethanol was sprayed all over the egg shells again sterilise the surface properly before further operation in a ventilated hood. Before opening the egg shells it is recommended to gently shake the eggs vertically to separate the embryos from their inner membranes. A small cut was created using a pair of sharp tweezers at the tropical circle of the eggs, then expanded the cut to about $2 \mathrm{~cm}$ with ophthalmic surgical scissors which allowed the shell to be opened manually to pour the five days old chicken embryos into a clean bowl with a diameter of $15 \mathrm{~cm}$ layered with clean polyethylene (PE) film.

Peking duck eggs (provided by Chinese University of Agriculture) with decent shape and symmetry were chosen and rinsed with tap water, then soaked in $0.1 \%$ benzalkonium chloride for $5-10$ minutes, dried and sterilised with $70 \%$ ethanol again. A PVC ring with the diameter as $4 \mathrm{~cm}$ was placed on the sharp end of the duck eggs, and a mark was labelled with a pencil. Then a dental drill with the blade diameter as $1 \mathrm{~cm}$ (Jinshida Equipment, China) was applied to open the eggs along the mark. The content of the eggs was poured out from the opening and this opening with the diameter as $4 \mathrm{~cm}$ would serve as a defined viewing window for the following observation. The prepared surrogate egg shells were further sterilised using PBS solution containing 1.60million units/L aminobenzyl penicillin combined with 2 million units/L streptomycin sulfate (P-S solution).

The chicken embryos extracted from the previous step were carefully transferred using the PE film layer into the surrogate duck egg shells. Now the film can be removed slowly from one corner and the position of the embryos can be adjusted to face upwards using a steel spoon, extra air bubbles should be carefully removed. A sterilised microenvironment for the repositioned embryos could be created by adding 5-6 drops of P-S solution along the rims of the egg shells. $1 \mathrm{~cm}$ nitrocellulose membranes (Merck Millipore) were soaked in advance with $10 \mu \mathrm{L}$ lipoprotein solutions with 
specified concentrations and placed carefully on the capillary beds of the chicken embryos. PE film discs with the diameter as $6 \mathrm{~cm}$ were cut and sealed onto the surrogate duck egg shells using the egg albumin solution left from the duck eggs, to avoid air leakage. The chicken embryos in duck egg shells were moved into the incubator at $370 \mathrm{c}$, humidity
$55-65 \%$, at $300 \mathrm{C}$ with a 2 hour turning over interval. Images of CAM were recorded using a Fujifilm digital camera after 48 and 72 hours. A summarised workflow is shown in Figure 1 .

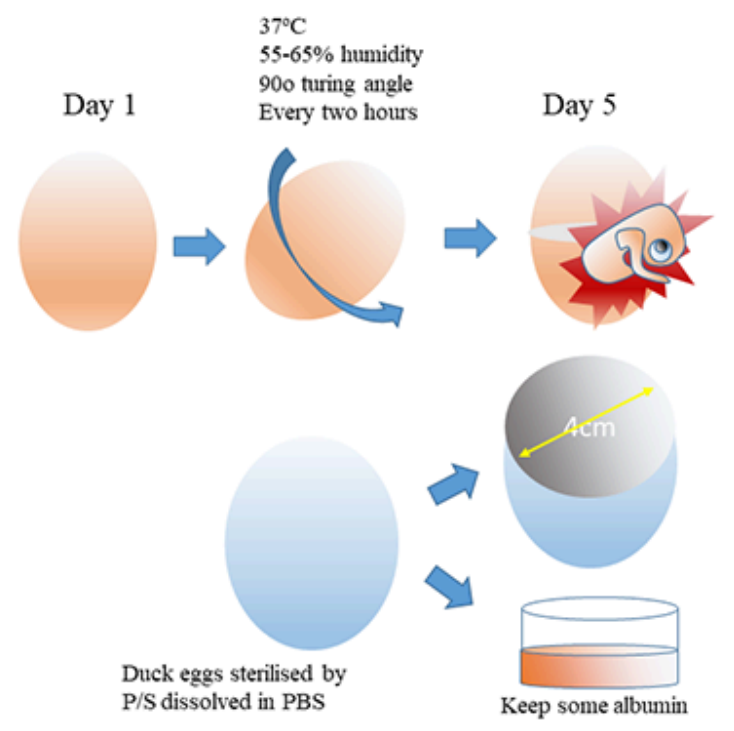

Film prepared to cover the opening

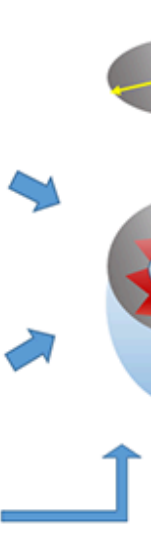

LDL
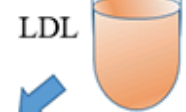

Chylomicrons

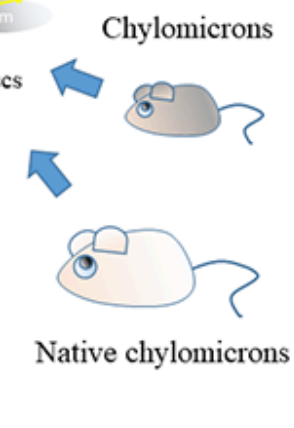

Native chylomicrons

Use some albumin

Filter paper discs Soaked with lipoproteins Residue to seal the opening

Figure 1

\section{Figure 1 caption}

Workflow of the improved CAM assay in surrogate egg shells; Fertilised chicken eggs were incubated for 5 days. A cut with a diameter around $2 \mathrm{~cm}$ was created with ophthalmic surgical scissors which allowed the shell to be opened manually to pour the chicken embryos for later use. Then surrogate shells were prepared and sterilised. A opening ring was defined with a PVC ring with the diameter as $4 \mathrm{~cm}$ on the sharp end of the surrogate eggs, then a dental drill was applied to open the eggs, and the content of the eggs was poured out from the opening, and this opening with the diameter as $4 \mathrm{~cm}$ would serve as a defined viewing window for the following observation. The chicken embryos extracted from the previous step were carefully transferred into the surrogate duck egg shells, and the position of the embryos can be adjusted to face upwards. Chylomicron solutions were prepared as described in Materials and Methods, and 10 $\mu \mathrm{L}$ lipoprotein solutions were absorbed into $6-\mathrm{mm}$ nitrocellulose membranes with specified concentrations, and concentrations and placed carefully on the capillary beds of the chicken embryos. After resealing the eggs with PE film discs using the egg albumin solution left from the duck eggs, air leakage. The chicken embryos in duck egg shells were moved into the incubator at 37 degrees, humidity $55-65 \%$, at 30 degrees with a 2 hour turning over interval. Images of CAM were recorded using a Fujifilm digital camera after 48 and 72 hours. * All the above operations were carried out in a ventilated hood; air leakage and air bubbles were minimised throughout the surgery process.

\section{Automated image analysis of CAM images}

The CAM angiogenesis assay images were analysed using a bespoke Fiji [16] macro. As these are natural images, taken on a Nikon camera, the analysis is not trivial with varying factors in each image such as uneven illumination as well as non-consistent 
image magnification and pixel size. The images also contain a date stamp which partially covers the region of interest and hence affects the analysis. The method for analysis is described below and we also make the macro available with this manuscript. Firstly, the date stamp is removed from the image. The date stamp in each image is overlayed by the camera hardware andas such it is of a consistent colour and can be extracted using the colour thresholding function in Fiji. Once extracted it is used to mask the date stamp in the original image. Secondly, the pixel size of an image is calibrated using the known diameter of the Peking duck egg shell within the image, $40 \mathrm{~mm}$. First the green component of the original image is extracted and smoothed to reduce any noise present in the image. A strong threshold is applied to extract the egg shell, which appears white in the green component image, and the maximum diameter of the segmented shell, in pixels, is used to calibrate the known distance $40 \mathrm{~mm}$. Also extracted are the approximate co-ordinates of the centre of the egg shell which are used in latter segmentation. Next the uneven illumination is corrected. The image is first split into its individual colour (RGB) components. Each of these component images is then smoothed to remove all features but leave an overall impression of the shading within the images, specifically we used a Gaussian kernel with a sigma of $0.5 \mathrm{~mm}$. These smoothed component images are then used to correct the shading in the original component images using the shading corrector plugin (https://imagej.nih.gov/ij/plugins/shadingcorrector.html). The corrected component images are then recomposed back into an RGB image.

To the image the Retinex algorithm [17] is now applied to enhance the vasculature in the embryo, which we are ultimately interested in, to allow for segmentation through simpler methods. After Retinex is applied the green component channel is once again extracted as it presents the best possibility for segmentation of the vasculature. To segment the vasculature multi-scale median filtering followed by thresholding was utilised. Using multi-scale filtering compensates for the variation in vessel diameter within each embryo. Each scale enhances vessels of that diameter allowing for segmentation of all vessels in the image. A maximum vessel diameter of $1 \mathrm{~mm}$, taken from multiple image observation, was employed. Finally, the centre co-ordinates of the egg shell, extracted in the image calibration step, along with the known diameter of the egg shell, are used to define a region of interest within the segmented image. This is to exclude any remaining background artefacts that may exist within the segmentation. The total vasculature area and length are extracted from the segmented image. The image analysis process is briefly summarised in Figure $\underline{2}$.

Statistics - Data are presented as mean \pm SEM, $\mathrm{n}=$ 3 independent experiments.
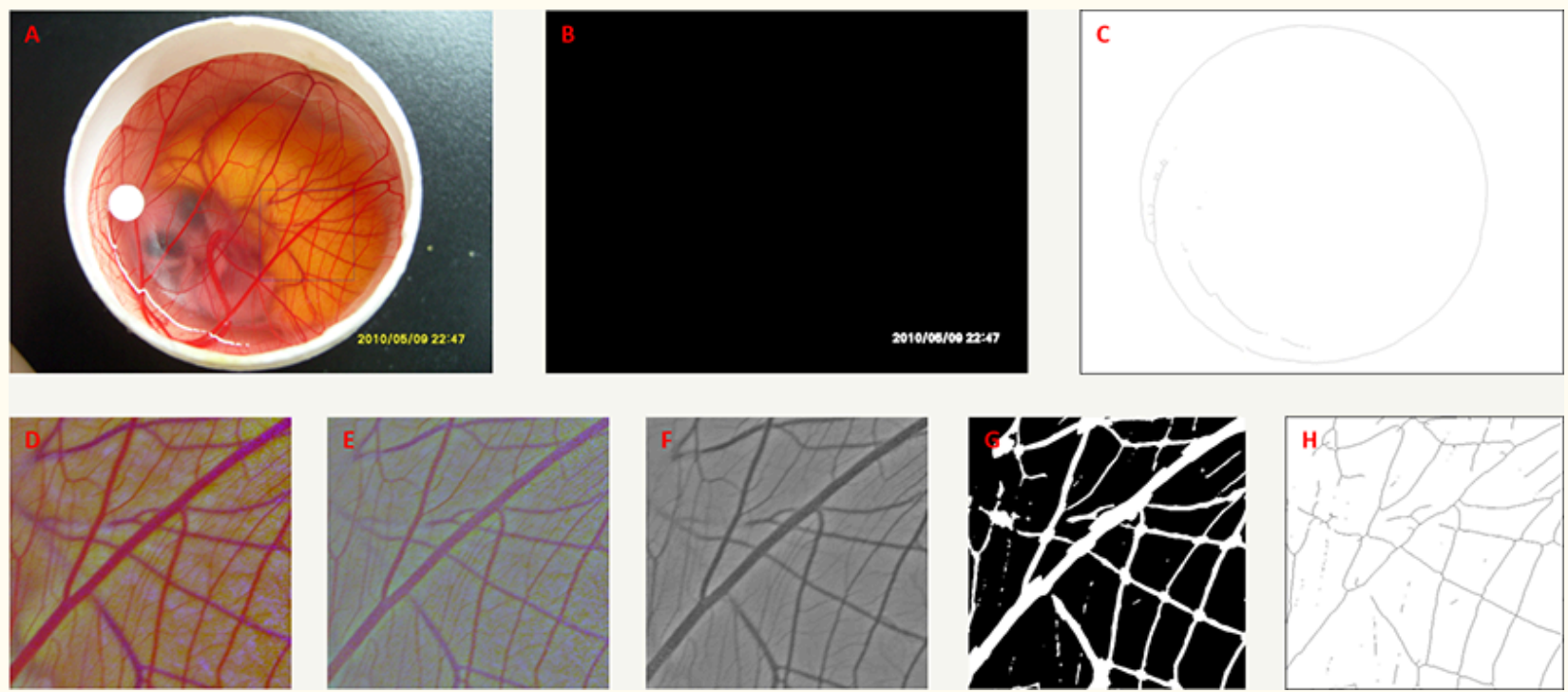

Figure 2 


\begin{abstract}
Figure 2 caption
Automated image analysis process for the improved CAM model. A) An example of an original input image. Square region marked is used in panels D to H. B) Time-stamp extraction using colour thresholding. C) Identification of the egg shell used to calibrate the pixel size in the images. D) Image post shading correction process. E) Result of Retinex processing to enhance vasculature. F) The green component of image post Retinex processing used for multi-scale median filtering and segmentation. G) Result of the segmentation process used to measure vasculature area. $\mathrm{H}$ ) Skeleton of segmentation used to measure vasculature length.
\end{abstract}

\section{Conclusions and Discussions}

The chicken embryo has the potential to become a classical model in basic and translational biology research, for instance, to study avian embryonic development or to model human physiological or pathological processes such as angiogenesis [18]. However, the limitations of operating directly on the eggs include damage to the embryos and limited viewing window areas. So far various artificial systems have been developed by other researchers, mainly based on plastic films or dishes [20]. Nevertheless, embryos relocated to artificial vessels face their own challenges aswell, including but not limited to, the material should preferentially be gas-permeable, such as polytetrafluoroethylene (PTFE), and the survival rate and health of the embryos suffers from the lack of physiologically relevant microenvironment factors such as sufficient calcium supply [18, 19]. Surrogate eggs have proven to be a plausible improvement provided that the physiological relevant development environment and barrier can be maintained by the surrogate shells [18]. Based on other researchers' approaches, we developed an improved the surrogate egg shell system using Peking duck egg shells to develop chicken embryos and defined a new technical details such as applying the original albumin solution from the surrogate eggs to seal up the opening to enhance the immunity integrity of the surrogate development vessels. In addition to the application described in this study, which is to model angiogenesis to evaluate effects of lipoproteins, this method can be further applied to a wider research community, such as to protect endangered avian species [20].

In this study, we used this optimized CAM model in a surrogate vessel, combining automated image analysis and revealed the regulating potential of chylomicrons on angiogenesis, for which there is still limited research. Studying the molecular link between chylomicrons and angiogenesis may help to understand the potential of modulating angiogenesis targeted therapy in dysfunctions relevant to metabolism such as atherosclerosis. Our research provides a new mechanism for the relationship between hypertriglyceridemia and atherosclerosis, by investigating the effects of CMs on angiogenesis, which is a risk factor of atherosclerosis [4]. Since angiogenesis can be beneficial in certain stages of plaque development by supplying nutrition for intima rebuilding, but can also increase the risk of fatal thrombosis, thus explaining the controversial roles of $\mathrm{CMs}$ in atherosclerosis development []].

The molecular mechanisms underlying the interaction of lipid metabolism and angiogenesis remain largely unknow. Other researchers pointed out the angiogenesis regulation effects by ApoBcontaining lipoproteins via VEGF receptor 1 modulation [21] and demonstrated that liver $X$ receptor activation reduces angiogenesis by impairing lipid raft localisation and signaling of vascular endothelial growth factor receptor - 2 [22]. Interestingly, both native and oxidative chylomicrons can increase the intracellular ROS in HUVECs and angiogenesis related parameters. However, at the same concentration, oxidized CMs enhanced angiogenesis more, suggesting oxidative modification still has effect on angiogenic regulation. Our research provides a new mechanism that hyperlipidemia interacts with angiogenesis and with roles of oxidative stress in this interaction. Other researchers have also shown that the oxidation of the metabolic product of lipids, fatty acid, is important for endothelial function [23]. However, how exactly fatty acid oxidation mediates angiogenesis remains undetermined. It is also noticeable that even angiogenesis has central roles in many physiological and pathological processes, the strategy of regulating angiogenesis still has its own limitations, including insufficient efficacy, refractoriness and tumour resistance $[\underline{4}, \underline{6}, \underline{12}, \underline{23}]$.

In conclusion, our study demonstrated the following advantages and application potentials of the improved CAM method. Firstly, the viewing window of CAM assay was improved in surrogate egg shells, allowing the automation of the image analysis pipeline. Secondly, the results of the analysis demonstrated the angiogenesis promoting effects of the oxidative LDL, which is consistent with other researchers' reports. Thirdly, the CAM model in surrogate shells combined with automated image analysis further revealed that chylomicrons, both native and potentially oxidised in the serum have pro-angiogenesis effects. Finally, HUVECs in vitro model suggested that oxidative stress may be 
involved in the promoting angiogenesis effects of chylomicrons.

\section{CMs increase angiogenesis in chicken chorioallantoic membrane (CAM) assay}

Both vasculature lengths and vasculature areas have been segmented and calculated using the automatic image analysis pipe-line described in Materials and Methods. As shown in Figure 3, both parameters showed a similar trend for the tested lipoproteins, suggesting the angiogenesis promoting potential of both native and oxidised chymomicrons at the tested concentrations. Interestingly, vasculature lengths revealed more significant effects compared to vasculature areas. For instance, the positive control ox-LDL showed as statistically different ( $p<0.05$ via T-test) from the control group when regarding to vasculature lengths, but no significant difference was shown between control group and ox-LDL treated group for vasculature area. For native chylomicrons directly extracted from SD rats, higher concentration $(300 \mu \mathrm{gTG} / \mathrm{mL})$ caused a more significant increase on vasculature lengths and areas than lower concentration $(150 \mu \mathrm{gTG} / \mathrm{mL})$, suggesting this angiogenesis promoting effect may be dependent on the dosage of triglyceride (TG). Nevertheless, for potentially oxidised chylomicrons extracted from GPIHBP1-/- mice showed a higher pro-angiogenic effect than ox-LDL and native chylomicron group at the concentration of $150 \mu \mathrm{g} \mathrm{TG} / \mathrm{mL}$, whereas a less significant pro-angiogenic effect was observed for the group treated with even higher concentration

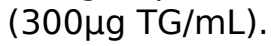

A
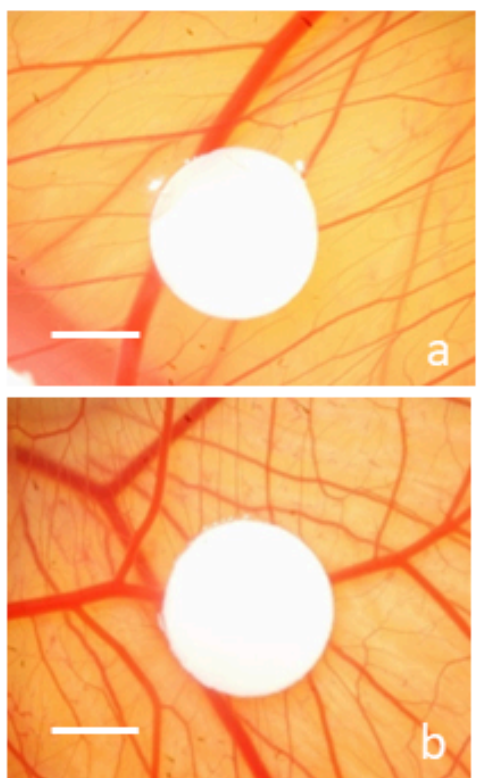

B

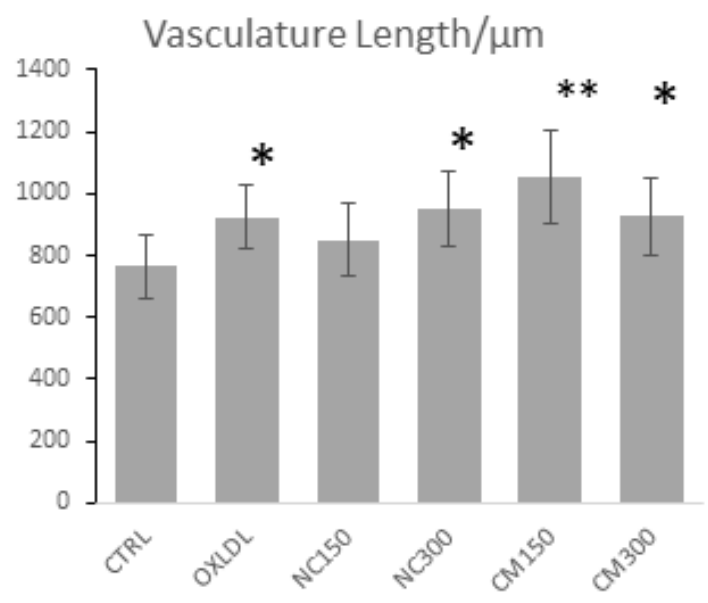

C

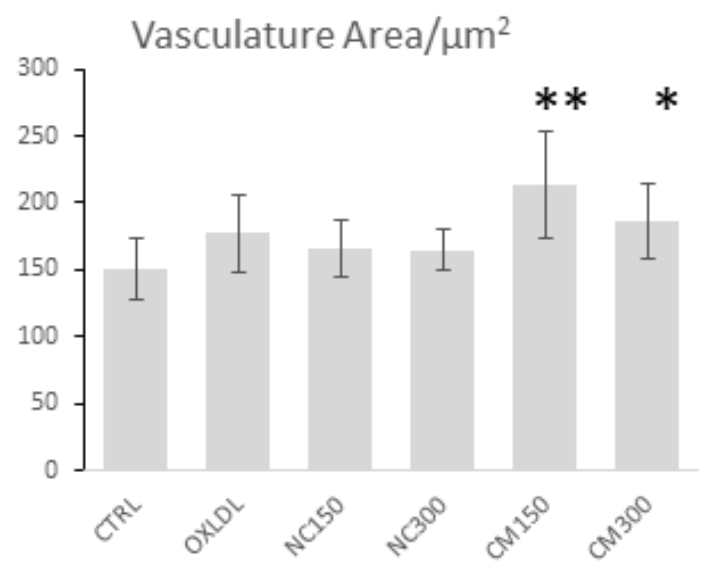

Figure 3
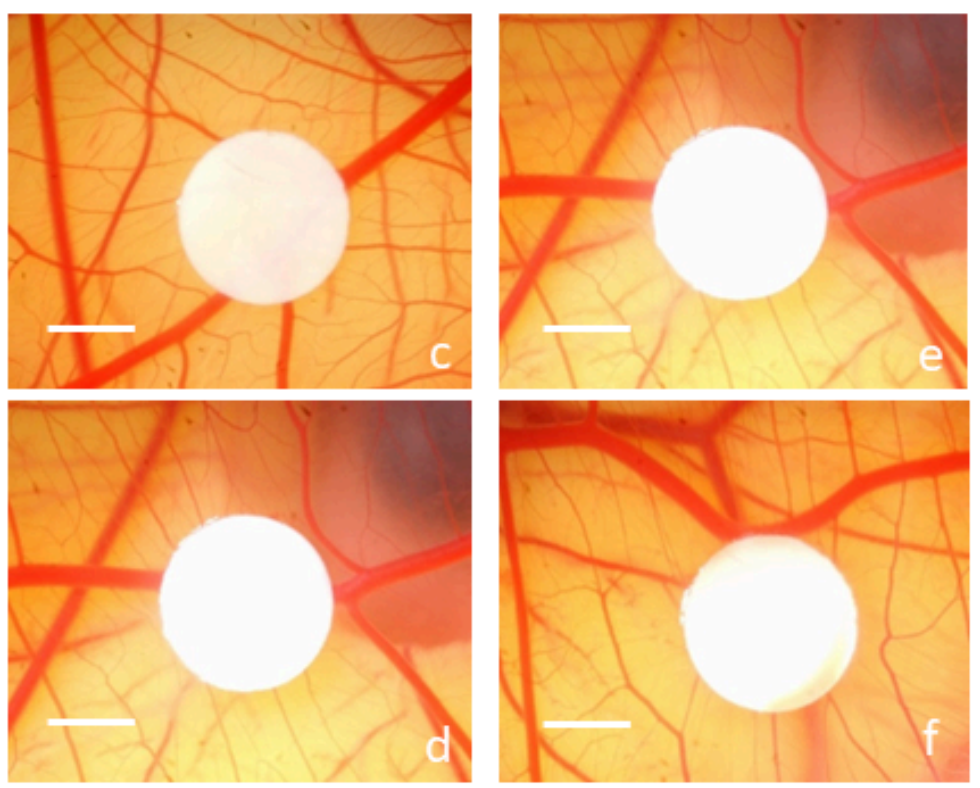


\section{Figure 3 caption}

CAM angiogenesis assay showing the pro-angiogenesis effects of the chylomicrons. (A) Representative images of CAM with different treatments. A. Ctrl; B. Ox-LDL 10 ugprotein /mL; C. NC 150 ug TG/mL; D. NC 300 ugTG/mL; E. CM 150 ugTG $/ \mathrm{mL} ;$ F. CM 300 ugTG $/ \mathrm{mL}$. Scale bar: $3 \mathrm{~mm}$. (B) Results of image analysis of the vasculature lengths of CAM models without or with chylomicron treatment. * $p<0.05$ compared with control group by Student's T-test. ** $p<0.01$ compared with control group based on Student's T-test; (C) Results of image analysis of the vasculature areas of CAMs without or with chylomicron treatment.

CMs increase the oxidative stress in in vitro human umbilical vein endothelial cells (HUVECs) in vitro model

We observed increased ROS and decreases NOS activity in HUVECS. Both native chylomicrons (NC) and chylomicrons (CM) increased DCF-sensitive intracellular ROS of HUVECs as shown in Figure 4. Under the same dosage, native CM-induced DCF fluorescence seems to be weaker than modified CM, and for each type of chylomicron, higher dosage caused more DCF-sensitive ROS generation. Ox-LDL was used as positive control. Generally, both native and modified chylomicrons have inducing effect on the proliferation of HUVECs. However, native chylomicrons have dose-dependent manner at 150 and $300 \mathrm{ugTG} / \mathrm{mL}$, while $150 \mathrm{ug} \mathrm{TG} / \mathrm{mL}$ modified chylomicrons have higher effect than the same dose of native ones, cytotoxicity was evident at 48 hours for $300 \mathrm{ugTG} / \mathrm{mL}$. The inducing effect of 150 ugTG/mL modified chylomicrons is similar to that of $300 \mathrm{ug} \mathrm{TG} / \mathrm{mL}$ native chylomicrons. It was also noticeable that CMs increased other angiogenesis related parameters in HUVECs, including cell proliferation (Supplementary Figure 2) and tubule formation (Supplementary Figure 3). Total nitric oxide synthase (NOS) activities were also inhibited by CMs treatments, served as another evidence for intracellular redox imbalance switch (Supplementary Figure 4). 
A
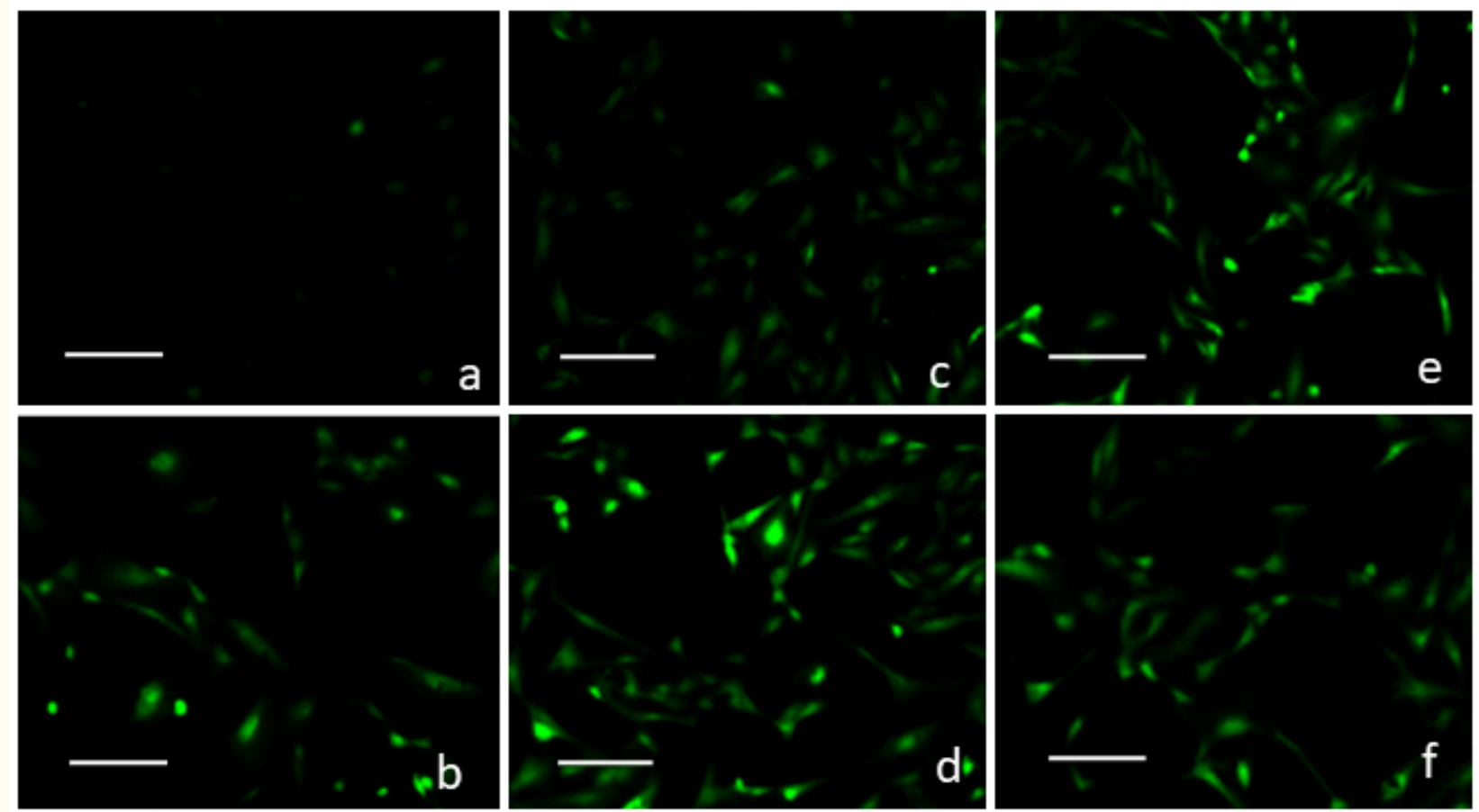

B

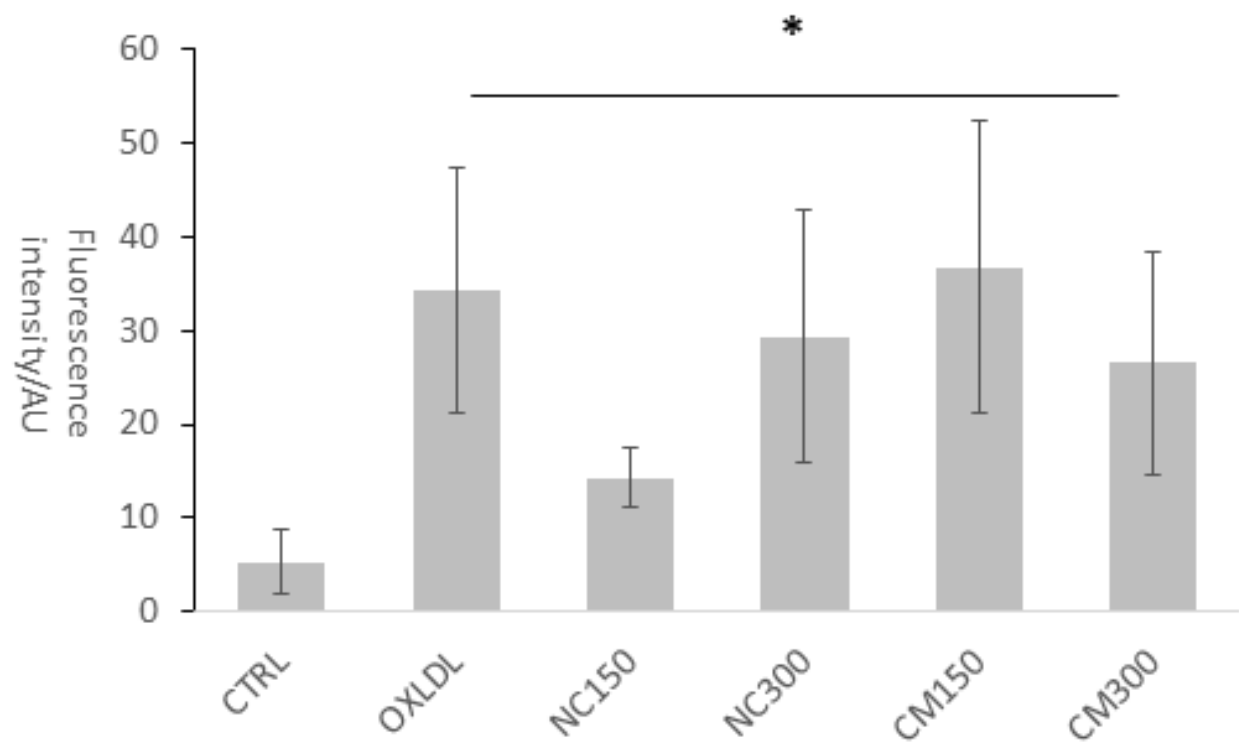

Figure 4

\section{Figure 4 caption}

CMs increased reactive oxygen species indicated by DCF fluorescence. (A) Representative DCF fluorescence microscopy images A. Ctrl; B. Ox-LDL 10ug protein $/ \mathrm{mL}$; C. NC 150 ug TG/mL; D. NC 300ug TG/mL; E. CM 150ug TG/mL; F. CM 300ug TG/mL. (B) Image analysis of DCF fluorescence images. $* p<0.05$ based on Student's T-test. 

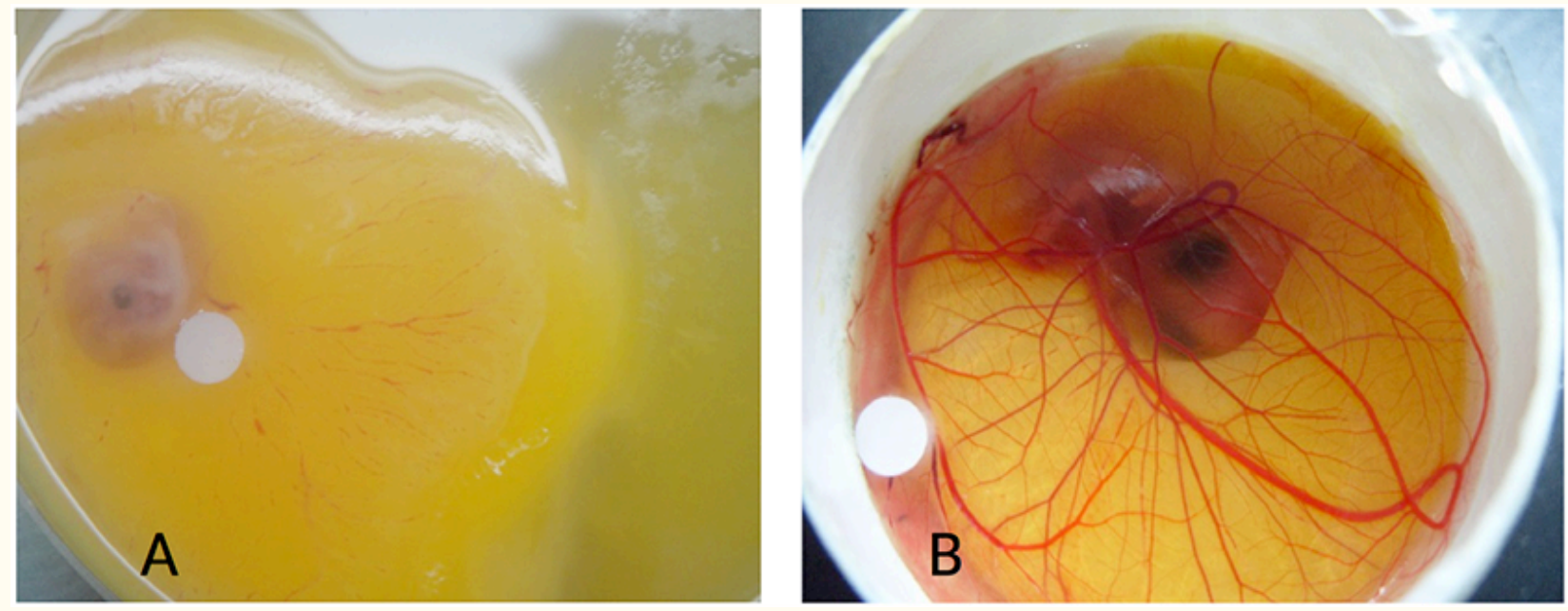

Supplementary Figure 1

\section{Supplementary Figure 1 caption}

Improved embryo viability of CAM assay in surrogate eggs: A. CAM assay in a petri dish with reduced viability; B. CAM assay in a surrogate egg shell with fully developed vasculature structure.
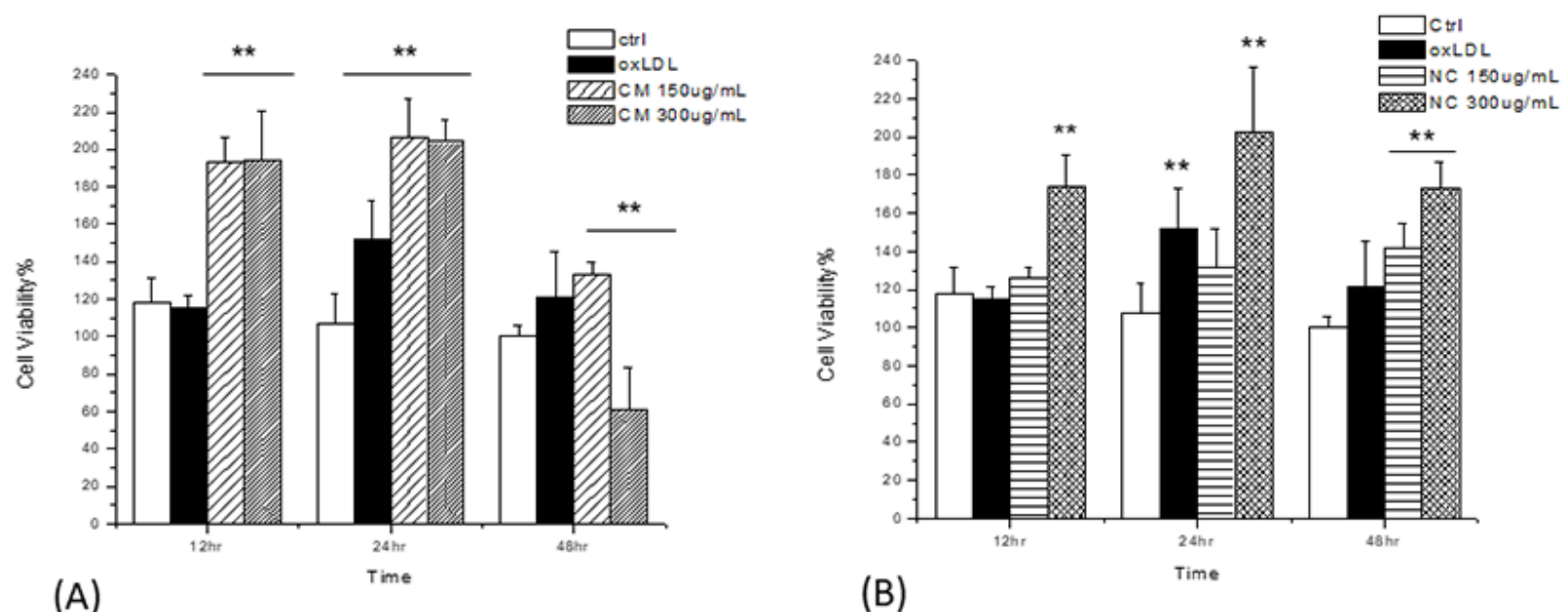

Supplementary Figure 2 


\section{Supplementary Figure 2 caption}

MTT assay (A) For modified chylomicrons, $150 \mathrm{ug} \mathrm{TG} / \mathrm{mL}$ has significant inducing effect similar to the effect of 300ug TG/mL native chylomicrons, while $300 \mathrm{ug} \mathrm{TG} / \mathrm{mL}$ modified $\mathrm{CM}$ has cytotoxic effect at 48 hours; (B) For native chylomicrons, $300 \mathrm{ug} \mathrm{TG} / \mathrm{mL}$ has more significant effect than 150 ug TG/mL.
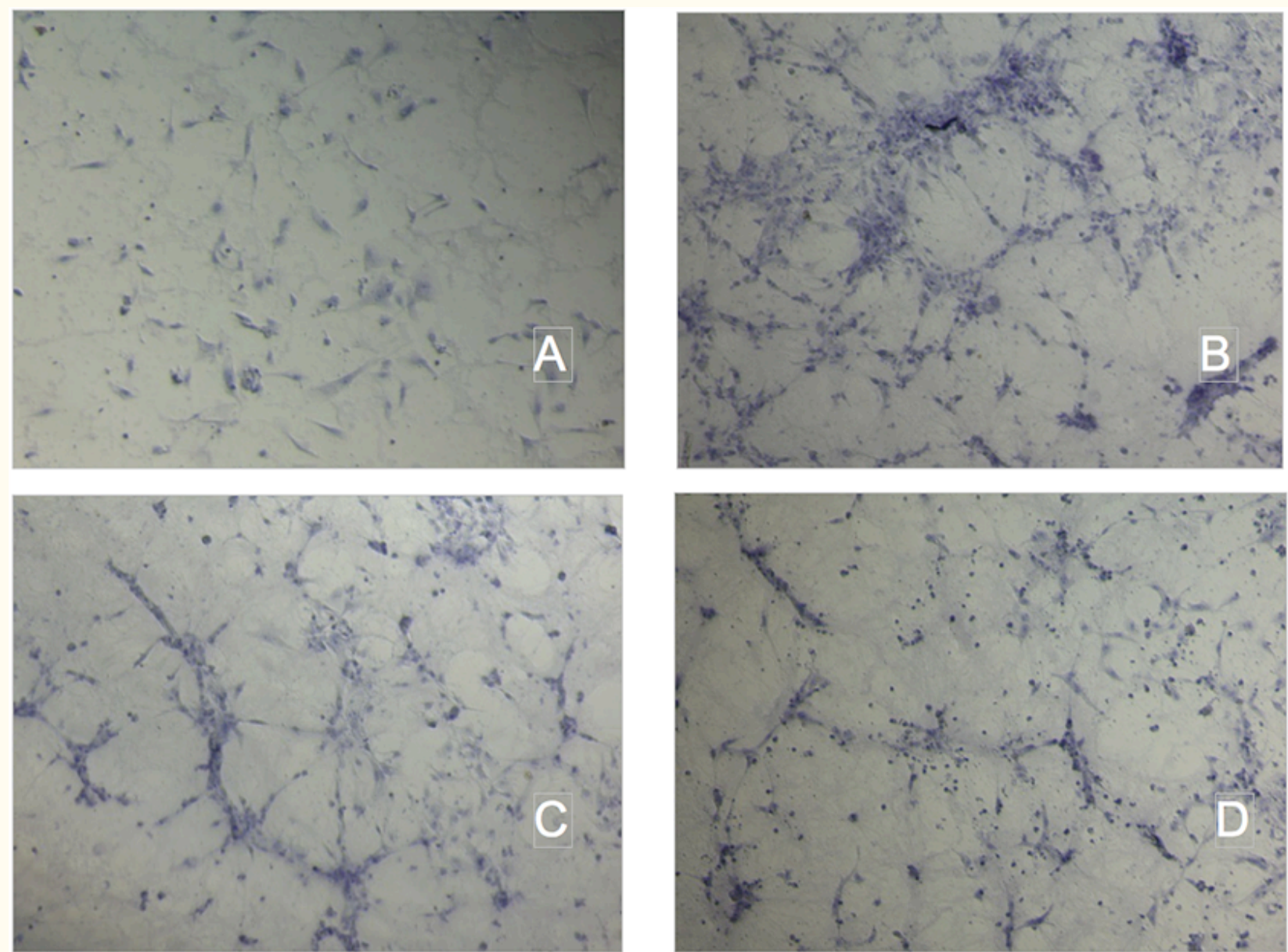

\section{Supplementary Figure 3}

\section{Supplementary Figure 3 caption}

Tube formation assay on Matrigel. A.Ctrl; B.Ox-LDL 10 ug protein/mL; C. NC 300ug TG/mL; D. CM 300ug TG/mL. 


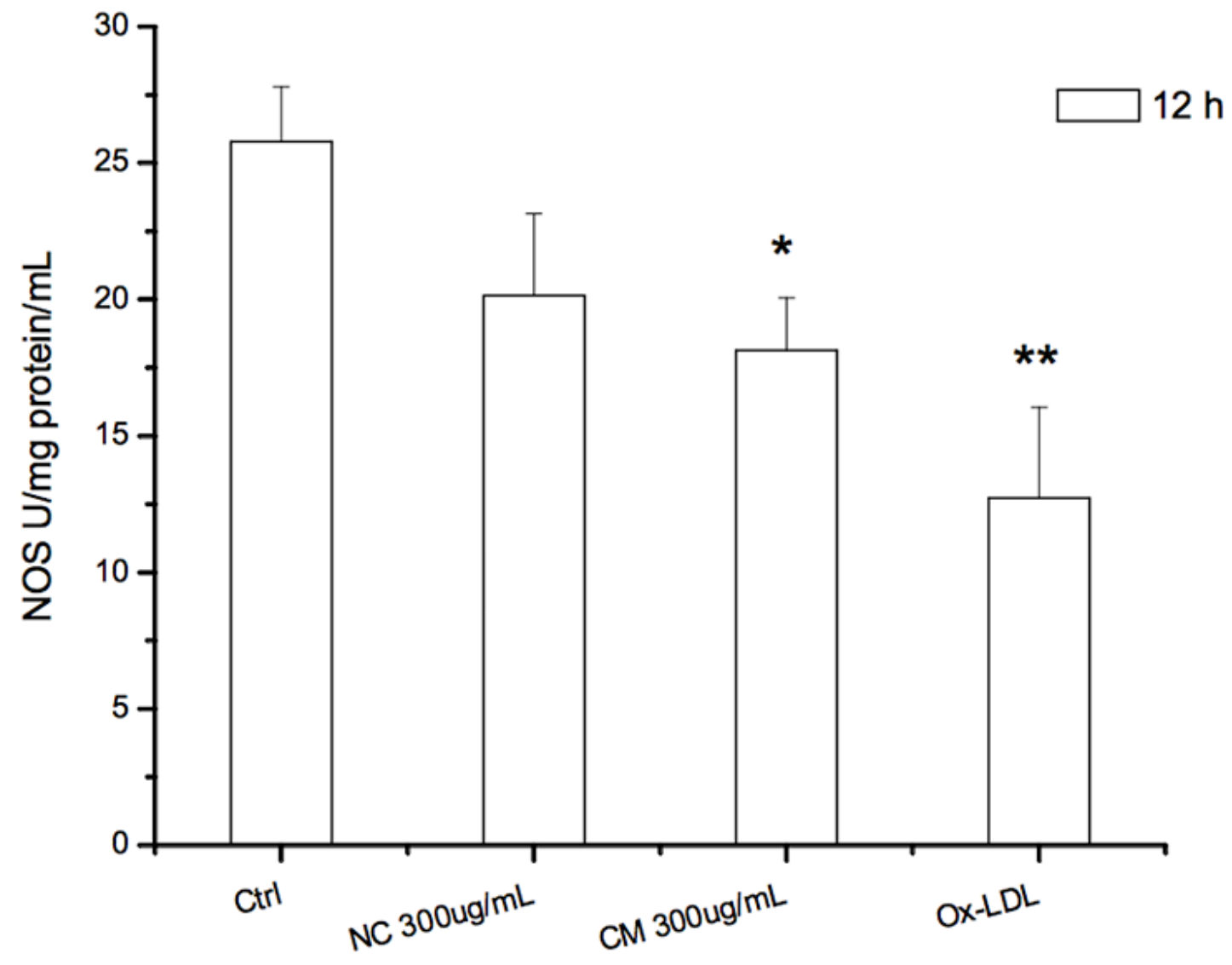

Supplementary Figure 4

Supplementary Figure 4 caption

NOS activities assay. $* p<0.05$ vs ctrl; $* * p<0.01$ vs ctrl.

\section{Acknowledgements}

\section{Equal contributions}

The authors Xiao Wan and Zifu Zhang contributed equally to the project.

\section{Funding disclaimer}

Xiao Wan is a Training Fellow funded by National Centre for the Replacement, Refinement $\&$ Reduction of Animals in Research (NC3Rs).

\section{Original submitted files for images}

Below are the links to the authors' original
Zifu Zhang is funded by Henan Province Key Technologies R\&D Program, Grant number 182300410028, 182106000049.

\section{Special thanks}

The authors would like to express their special thanks to Professor George Liu and Dr Yuhui Wang in Peking University Institute of Cardiovascular Sciences, for their generous support and kind advice on the experiments, data management and final drafting of the manuscript.

submitted files for images. 
Original image file for Figure 1

Click here to view.

Original image file for Figure 2

Click here to view.

Original image file for Figure 3

Click here to view.

Original image file for Figure 4

Click here to view.
Original image file for Supplementary Figure 1

Click here to view.

Original image file for Supplementary Figure 2

Click here to view.

Original image file for Supplementary Figure 3

Click here to view.

Original image file for Supplementary Figure 4 Click here to view.

\section{References}

1. Nowak-Sliwinska P, Segura T, Iruela-Arispe ML. The chicken chorioallantoic membrane model in biology, medicine and bioengineering.

Angiogenesis. 2014;4:779-804.

2. Moreno-Jiménez I, Hulsart-Billstrom $G$, Lanham SA, Janeczek AA, Kontouli N, Kanczler JM, Evans ND, Oreffo R. The chorioallantoic membrane (CAM) 12 assay for the study of human bone regeneration: a refinement animal model for tissue engineering. Sci Rep. 2016;6:32168-.

3. Ono T, Murakami T, Mochii M, Agata K, Kino K, Otsuka K, Ohta M, Mizutani M, Yoshida M, Eguchi G. A complete culture system for avian transgenesis, supporting quail embryos from the single-cell stage to hatching. Dev

Biol. 1994;1:126-30.

4. Carmeliet $P$, Jain R. Angiogenesis in cancer and other diseases. Nature. 2000;407:249-257.

5. Chwalek K, Bray LJ, Werner C. Tissue-engineered 3D tumor angiogenesis models: Potential technologies for anti-cancer drug discovery. Adv Drug Deliv Rev. 2014;79:30-39.

6. Slevin M, Krupinski J, Badimon L. Controlling the angiogenic switch in developing atherosclerotic plaques: Possible targets for therapeutic intervention. J Angiogenesis Res. 2009;1:4-.

7. Hussain MM. Review Article: A proposed model for ${ }^{16}$ the assembly of chylomicrons.

Arterosclerosis. 2000;148:1-15.

8. Calle EE, Rodriguez C, Walker-Thurmond K, Thun MJ. Overweight, obesity, and mortality from cancer in a prospectively studied cohort of U.S. adults. N Engl J Med. 2003;17:1625-38.

9. Menendez JA, Lupu R. Fatty acid synthase and the lipogenic phenotype in cancer pathogenesis. Nat Rev Cancer. 2007;10:763-77.

10. Iwamoto $H$, Abe $M$, Yang $Y$, Cui $D$, Seki $T$, Nakamura M, Hosaka K, Lim S, Wu J, He X, Sun X, ${ }^{19}$ Lu Y, Zhou Q, Shi W, Torimura T, Nie G, Li Q, Cao Y. Cancer lipid metabolism confers antiangiogenic drug resistance. Cell Metabolism. 2018;1:104-117 20.
11. Zhang X, Qi R, Xian X, Yang F, Blackstein M, Deng $X$, Fan J, Ross C, Karasinska J, Hayden MR, Liu G. Spontaneous atherosclerosis in aged lipoprotein lipase-deficient mice with severe hypertriglyceridemia on a normal chow diet. Circ Res. 2008;102:250-256.

12. Nordestgaard BG, Tybjaerg-Hansen A. IDL, VLDL, chylomicrons and atherosclerosis. Eur J

Epidemiol. 1992;1:92-98.

13. Yu S, Wong SL, Lau CW, Huang Y, Yu CM. Oxidized LDL at low concentration promotes in-vitro angiogenesis and activates nitric oxide synthase through PI3K/Akt/eNOS pathway in human coronary artery endothelial cells. Biochem Biophys Res Commun. 2011;1:44-48.

14. Wan X, Gou B, Wang K. Gadolinium-promoted angiogenesis involves the activation of $P K C \alpha / \beta 2$ and MAPKs in human umbilical vein endothelial cells. J Chinese Pharm Sci. 2013;1:71-76.

15. Li H, Han $Y$, Qi R, Wang $Y$, Zhang $X$, Yu M, Tang $Y$, Wang $M$, Shu $Y N$, Huang $W$, Liu $X$, Rodrigues $B$, Han M, Liu G. Aggravated restenosis and atherogenesis in ApoCIII transgenic mice but lack of protection in ApoCIII knockouts: the effect of authentic triglyceride-rich lipoproteins with and without ApoCIII. Cardiovasc Res. 2015;4:579-89.

Schindelin J, Arganda-Carreras I, Frise E, Kaynig V, Longair M, Pietzsch T, Preibisch S, Rueden C, Saalfeld S, Schmid B, Tinevez JY, White DJ, Hartenstein V, Eliceiri K, Tomancak P, Cardona A. Fiji: an open-source platform for biological-image analysis. Nat Methods. 2012;7:676-682.

7. Land EH. The Retinex theory of color vision. Sci Am. Dec 1997;237(6):108-128.

8. Borwompinyo S, Brake J, Mozdziak PE, Petitte JN. Culture of chicken embryos in surrogate eggshells. Poult Sci. 2005;9:1477-82.

19. Tahara Y, Obara K. A Novel shell-less culture system for chick embryos using a plastic film as culture vessels, J. Poult. Sci. 2014;51:307-312.

Liu C, Jörg K, Vijay B, Zhor G, Ulrich W, II-Kuk C. Houbara bustard hatching in surrogate chicken 
albumen and eggshell. Avian Bio

Res. 2013;3:239-242.

21. Avraham-Davidi I, Ely Y, Pham VN, Castranova D, Grunspan M, Malkinson G, Gibbs-Bar L,

Mayseless O, Allmog G, Lo B, Warren CM, Chen TT, Ungos J, Kidd K, Shaw K, Rogachev I, Wan W, Murphy PM, Farber SA, Carmel L, Shelness GS, Iruela-Arispe ML, Weinstein BM, Yaniv K. ApoBcontaining lipoproteins regulate angiogenesis by modulating expression of VEGF receptor 1 . Nat Med. 2012;6:967-73.

\section{Copyright \& License}

Statement: Copyright $\odot$ 2019, Xiao Wan, Zifu Zhang, Lei Rui, Rhodri S. Wilson, Xiaoning Wu, Xin Liu. Holder: Xiao Wan, Zifu Zhang, Lei Rui, Rhodri S. Wilson, Xiaoning Wu, Xin Liu

Licensee: Publiverse Online S.R.L.

License: Open Access This article is distributed under the terms of the Creative Commons Attribution 4.0 International License (http://creativecommons.org/licenses/by/4.0/), which permits unrestricted use, distribution, and reproduction in any medium, provided you give appropriate credit to the original author(s) and the source, provide a link to the Creative Commons license, and indicate if changes were made. The Creative Commons Public Domain Dedication waiver (http://creativecommons.org/ publicdomain/zero/1.0/) applies to the data made available in this article, unless otherwise stated.

\section{VascularCell A PUBLyERSE A ON!JNE}

The present article has been published in Vascular Cell journal by Publiverse Online S.R.L. 